\title{
Choristoneura fumiferana Granulovirus pk-1: A Baculoviral Protein Kinase
}

\author{
Paresa N. Giannopoulos ${ }^{\dagger}$ Nasha Nassoury ${ }^{\ddagger}$, Lucie Lamontagne ${ }^{\S}$, Claude Guertin ${ }^{\dagger}$ and Kianoush Khajeh Rashidan ${ }^{\S, *}$ \\ "Institut national de la recherche scientifique-Institut Armand-Frappier 531 Blvd des Prairies, H7V 1B7, Laval, Canada \\ Université de Montréal, 4101 Sherbrook east, H1X 2B2 Montreal, Canada \\ ${ }^{\S}$ Université du Québec à Montréal, Succursal Centre Ville CP8888, H3C 3P8 Montreal, Canada
}

Received 4 December 2004, Accepted 31 March 2005

\begin{abstract}
Open reading frame (ORF) 3 on the Choristoneura fumiferana granulovirus (ChfuGV), located in the $11 \mathrm{~kb}$ fragment of the BamHI genomic bank encodes a predicted 32-kDa putative kinase protein. Bioinformatics analysis on the predicted amino acid sequence of ChfuGV PK-1 revealed the existence of 11 catalytic subdomains. Sequence analysis within the 5'-untranslated region (5'-UTR) of ChfuGV pk1 indicates the presence of both putative early and late promoter motifs, indicating that $p k-1$ may be expressed throughout the infection cycle. Promoter sequence analysis reveals that $p k-1$ is deprived of a TATA box and appears instead to be regulated by other cis-acting transcriptional regulatory elements. Temporal transcription analysis by RT-PCR confirms the appearance of transcripts detected from $2 \mathrm{~h}$ p.i. until $72 \mathrm{~h}$ p.i. Northern blot hybridization characterizes $p k-1$ transcription as a $1.2 \mathrm{~kb}$ transcript. Homology comparisons reveal that ChfuGV PK-1 protein is most closely related to Phthorimaea operculalla granulovirus (PoGV) with $80 \%$ amino acid identity.
\end{abstract}

Keywords: Apoptosis, Baculovirus, Catalytic domain, Choristoneura fumiferana granulovirus, Kinase protein, Protein analysis, Transcription

\section{Introduction}

Choristoneura fumiferana granulovirus (ChfuGV), a virus belonging to the family Baculoviridae, is pathogenic towards spruce budworm (Choristoneura fumiferana, (Clem.), (Lepidoptera: Tortricidae)). Baculoviruses are divided into two genera, nucleopolyhedrovirus (NPV) and granulovirus (GV) (Blissard and Rohrmann, 1990; Rohrmann, 1999; Winstanley and O'Reilly, 1999). ChfuGV is characterized by rod-shaped

\footnotetext{
* To whom correspondence should be addressed.

Tel: 514-987-3000; Fax: 514-987-4647

E-mail: kiarashidan@juno.com
}

enveloped virions containing supercoiled double-stranded circular DNA genomes. Unlike NPVs, GVs are more difficult to grow in cell cultures (Winstanley and Crook, 1993), therefore limited molecular and biochemical information is available.

The life cycle of baculoviruses includes two genetically identical, but morphologically and temporally distinct forms of infectious progeny: an occluded derived virion (ODV) and budded virion (BV) (Blissard et al., 2000). The viral structure consists of a nucleocapsid, a lipid membrane envelope, the latter differing in source and composition for each phenotype (Braunagel and Summers, 1994). It is still unknown what causes these differences at the molecular level. The ODV form is also embedded in the protective occlusion body, composed primarily of the matrix protein granulin, in the case of granuloviruses (GVs). ODVs of GVs usually contain a single virion, in contrast to NPVs (Winstanley and O'Reilly, 1999). The viral cycle of infection begins by ODV ingestion, followed by loss of the occlusion body due to environmental alkaline $\mathrm{pH}$ and fusion into host's midgut epithelial cells (Wang et al., 1997). Once released into the nucleus, viral transcription and DNA replication subsequently occur. Early after infection, the virus buds through the plasma membrane to obtain its envelope resulting in the BV form and spreads systemically through the endocytic pathway towards other types of cells within the same host (Jarvis and Garcia, 1994). ODVs appear abundantly only at late times post-infection causing cell lysis and viral transmission to other larvae.

There are three major phases of gene expression, namely early, late and very late, controlled in a regulatory cascade manner (Friesen, 1997; Lu and Miller, 1997). All genes are located randomly throughout the genome, even though their products fill distinct regulatory or structural functions. Early expression is in the absence of de novo viral protein synthesis whereas late and very late gene expression requires RNA polymerase composed of virus-encoded subunits (Guarino et al., 1998). The infection cycle of viruses may sometimes be complex. Several regulatory enzymatic proteins, such as kinase proteins, may be implicated to ensure many of their activities. 
Protein phosphorylation was first identified as a mechanism for regulating protein activity in the 1950's (Fischer and Krebs, 1955) and is still considered as one of the most important regulatory mechanisms which is mediated by kinase proteins for enabling a cell to respond biologically to external stimuli. In addition, kinase proteins are able to phosphorylate via their conserved structural catalytic domains through which they bind both ATP and peptide substrate before catalyzing the transfer of a phosphoryl group to a substrate's hydroxyl group on either Ser/Thr or Tyr residues (Hanks and Hunter, 1995). They regulate a wide range of eukaryotic cellular functions, including transcription, translation, cell division and differentiation (Graves and Krebs, 1999; Hunter, 2000) leading them to represent one of the largest superfamilies of proteins known. In fact, protein kinase domains are found in approximately $2 \%$ of eukaryotic genes (Rubin et al., 2000). Hundreds of kinase proteins have been discovered in various species including viruses (Leader, 1993). Protein phosphorylation has also been discovered in baculoviruses. Among the several phosphoproteins identified to date, included are: pp31 (Guarino et al., 1992), gp64 (Maruniak and Summers, 1981), pp34 (Whitt and Manning, 1988), p78/83 (Vialard and Richardson, 1993), IE-1 (Choi and Guarino, 1995), and p6.9 (Kelly and Lescott, 1984). In addition, phosphorylation has been shown to occur on both non-occluded and occluded forms of AcMNPV (Miller et al., 1983), in nucleocapsids of PiGV (Wilson and Consigli, 1985) and within silkworms infected with BmNPV (Zemskov et al., 1992). All these protein kinase activities have not yet been associated to any baculovirus-encoded kinase protein. Only a few baculoviruses have been proven to encode an actual kinase protein (Bischoff and Slavicek, 1994; Reilly and Guarino, 1994; Li and Miller, 1995; Zhang et al., 1998). The functional roles of these baculoviral-encoded kinase proteins remain to be assigned.

Here we report the identification and characterization of $p k-1$ gene on ChfuGV genome. The conserved kinase catalytic subdomains were revealed. All cis-acting elements within the 5 '-UTR of the promoter of ChfuGV pk-1 were located. The temporal transcriptional pattern for ChfuGV $p k-1$ was analyzed using Reverse Transcriptase-PCR (RT-PCR). The presence and the sizes of $p k-1$ transcripts were determined by Northern blot hybridization. The phylogenetic relatedness of PK-1 to baculoviral kinases and other kinases was also demonstrated.

\section{Materials and Methods}

Viral propagation, DNA extraction, cloning, and sequencing The virus was produced in vivo by a method described previously (Rashidan et al., 2002). ChfuGV genomic DNA was extracted from pure enveloped nucleocapsids, cloned and sequenced as explained before (Rashidan et al., 2002).

RNA isolation and quantification Infected larvae were harvested at $0,2,6,12,24,48,72$, and 96 hours post-infection (h p.i.). A negative control of non-infected larvae was also prepared. Time zero was defined at the end of the $10 \mathrm{~min}$ inoculation/absorption period. Total RNA was isolated using TRIzol ${ }^{\circledR}$ (Invitrogen, Life technologies, San Diego, USA), an acid phenol extraction reagent containing detergents and guanidine thiocyanate to denature RNAses, based on the procedure previously described (Chomczynski and Sacchi, 1987). Total RNA was suspended in DEPC-treated water and stored in aliquots at $-70^{\circ} \mathrm{C}$. Samples were quantified spectrophotometrically.

RT-PCR and Northern blot analyses The integrity of total RNA was verified after electrophoresis through $1 \%$ agarose gel. The presence of DNA was verified by PCR. A one-step RT-PCR reaction was performed to detect $p k-1$ mRNA (Invitrogen, Life technologies). Total reaction mixture was $50 \mu \mathrm{L}$, which included 1 $\mu \mathrm{g}$ of total RNA, 150-172 mmoles of forward and reverse $p k-1$ primers (pkfw1 5'-CCATTGGAGTTCAGCGTTGC-3' and pkrv1 5'-CATCATAACTGCTGAGGC-3'), appropriate RT (Superscript II, MMLV)/Plat Taq enzyme mix, and $1 \mathrm{X}$ manufacturer's reaction buffer, containing $0.2 \mathrm{mM} \mathrm{dNTP}$ and $1.2 \mathrm{mM} \mathrm{MgSO}_{4}$. The first strand cDNA was synthesized in a single cycle of $50^{\circ} \mathrm{C}$ for $30 \mathrm{~min}$, followed by a pre-denaturation of $2 \mathrm{~min}$ at $94^{\circ} \mathrm{C}$. cDNA was used as template for subsequent PCR amplification. Using the GeneAmp ${ }^{\circledR}$ PCR System 9700 (Applied Biosystems, Foster City, USA), a PCR reaction of 35 cycles contained the following temperatures: $94^{\circ} \mathrm{C}$ for $30 \mathrm{~s}, 50^{\circ} \mathrm{C}$ for $30 \mathrm{~s}$, and $72^{\circ} \mathrm{C}$ for $45 \mathrm{~s}$. After the final cycle, the temperature was held at $72^{\circ} \mathrm{C}$ for $10 \mathrm{~min}$.

RNAs were size-fractionated on MOPS-formaldehyde agarose $(1.2 \% \mathrm{w} / \mathrm{v})$ gels and capillary transferred to positively charged nylon membrane (Hybond $^{\mathrm{TM}}$, Amersham Biosciences, Richmond, USA) with 20X SSC. The membrane was baked for $30 \mathrm{~min}$, UVcrosslinked and prehybridized at $65^{\circ} \mathrm{C}$ for $1 \mathrm{~h}$ in buffer solution composed of $5 \mathrm{X}$ SSC, $5 \mathrm{X}$ Denhardt's solution, $0.5 \%$ SDS, and 50 $\mu \mathrm{g} / \mathrm{mL}$ heat-denatured salmon sperm DNA. Hybridization was then carried out overnight at $65^{\circ} \mathrm{C}$ with $\left[\alpha-{ }^{32} \mathrm{P}\right] \mathrm{dCTP}(3000 \mathrm{Ci} / \mathrm{mmol}$, ICN) labeled DNA probe prepared by random priming using the PCR amplicon of $p k-1$ (Ready-To-Go ${ }^{\mathrm{TM}}$, Pharmacia Biotech, Uppsala, Sweden). The blot was washed twice in $2 \mathrm{X}$ SSC, $0.1 \%$ SDS and in $1 \mathrm{X}$ SSC, $0.1 \%$ SDS. Exposures were made on Kodak $\mathrm{X}$-ray film (X-OMAT ${ }^{\mathrm{TM}}$ Blue $\left.\mathrm{XB}-1\right)$ at $-80^{\circ} \mathrm{C}$ using intensifying screens before being subjected to autoradiography.

Computer analysis The sequences were analyzed by Sequencer program version 4.0.5 (Gene Codes Corp.) and MacVector program version 4.5.0. (Eastman Kodak). The nucleotide sequence and its predicted amino acid sequence were compared to homologues in GenBank/EMBL and SWISSPROT by using BLAST algorithm (Altshul et al., 1992). CLUSTALX (1.81) (Thompson et al., 1997) was used for multiple amino acid sequence alignments. BOXSHADE was used for similarity shading and scoring among the aligned sequences. Post-translational modifications were predicted using ProtParam (http://www.expasy.ch/tools/protparam.html).

\section{Results and Discussion}

Nucleotide sequence analysis The $p k-1$ gene of ChfuGV was found within the $11 \mathrm{~kb}$ fragment of the BamHI genomic 


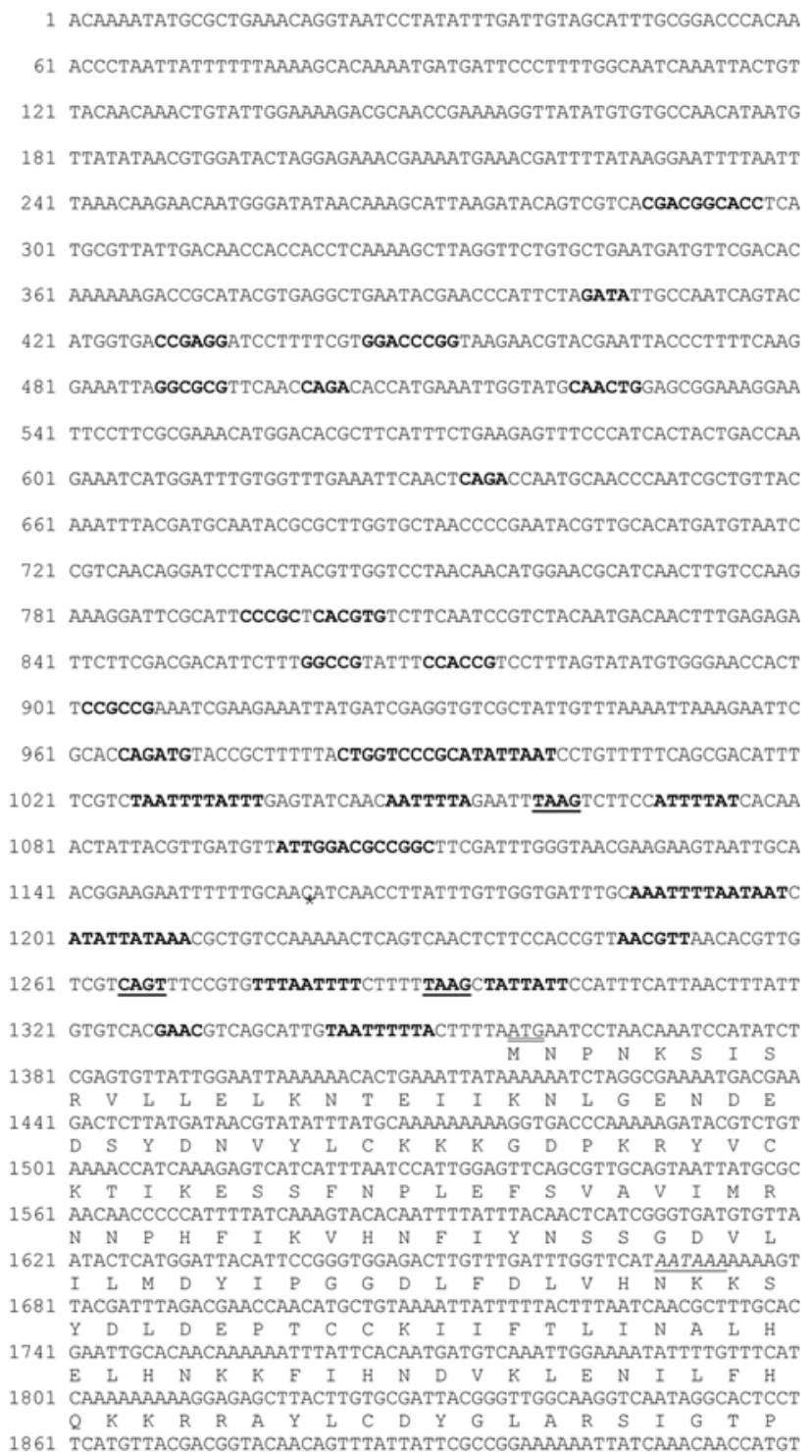

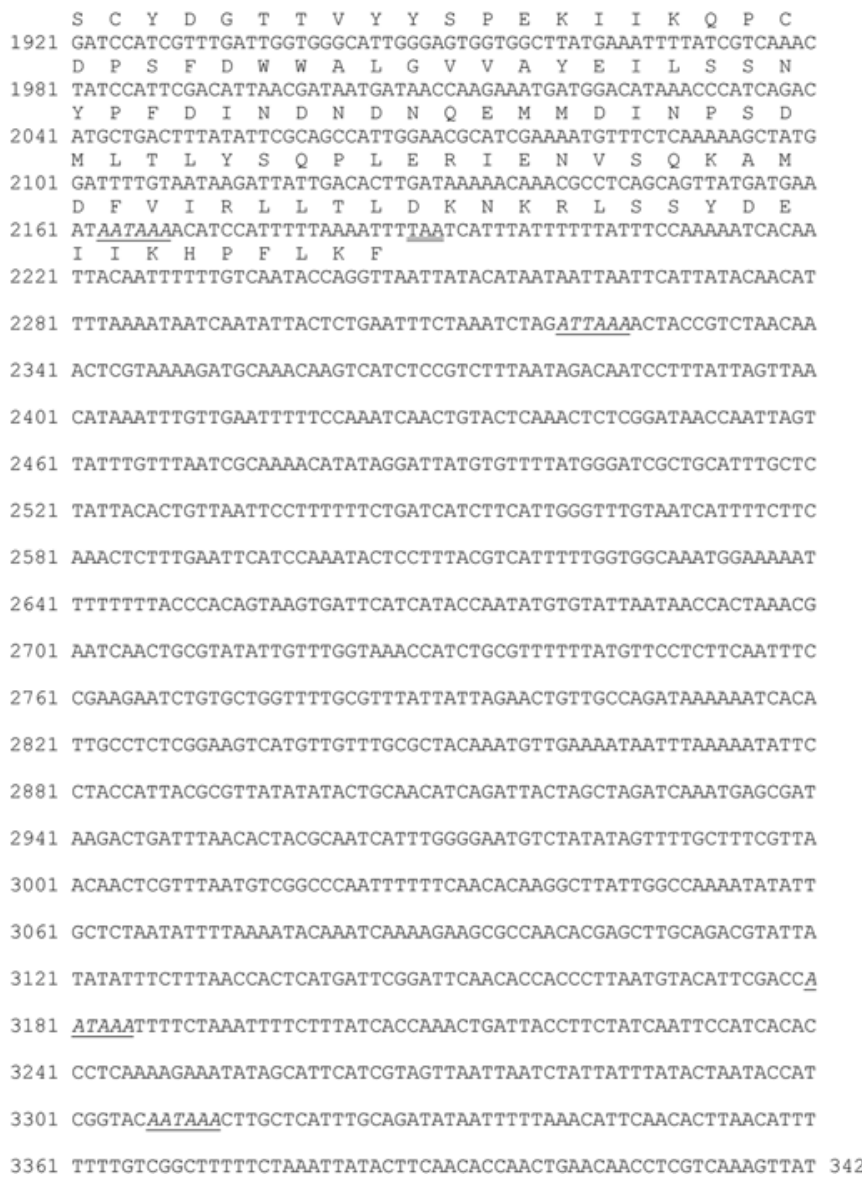

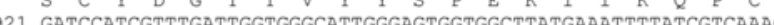
$\begin{array}{lllllllllllllllllllll}D & P & S & E & D & W & W & A & L & G & V & V & A & Y & E & I & L & S & S & N\end{array}$ A

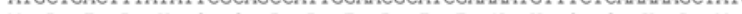
$M$ L $T$ L Y S Q P L E R I E $N$ $V$ S Q K A M $\begin{array}{llllllllllllllllllll}\text { D } & \text { F } & \text { V } & \text { I } & \text { R } & \text { L } & \text { L } & \text { T } & \text { L } & \text { D } & \text { K } & \text { N } & K & \text { R } & \text { L } & \text { S } & \text { S } & \text { Y } & \text { D } & \text { E }\end{array}$ 1 AT AATAAAACATCCATTTTAAAAATTTTAATCATTTATTTTTATTTCCAAAAATCACAA 1 TTACAATTTTTTGTCAATACCAGGTTAATTATACATAATAATTAATTCATTATACAACAT 2281 TTTAAAATAATCAATATTACTCTGAATTTCTAAATCTAGATTAAAACTACCGTCTAACAA 2341 ACTCGTAAAAGATGCAAACAAGTCATCTCCGTCTTTAATAGACAATCCTTTATTAGTTAA 2401 CATAAATTTGTTGAATTTTTCCAAATCAACTGTACTCAAACTCTCGGATAACCAATTAGT 2461 TATTTGTTTAATCGCAAAACATATAGGATTATGTGTTTTATGGGATCGCTGCATTTGCTC 2521 TATTACACTGTTAATTCCTTTTTTCTGATCATCTTCATTGGGTTTGTAATCATTTTCTTC 2581 AAACTCTTTGAATTCATCCAAATACTCCTTTACGTCATTTTTGGTGGCAAATGGAAAAAT 2641 TTTTTTTACCCACAGTAAGTGATTCATCATACCAATATGTGTATTAATAACCACTAAACG 2821 TTGCCTCTCGGAGTCATGTTGTTTGCGCTACAAATGTTGAAAATAATTTAAAAATATTC 3001 ACAACTCGTTTAATGTCGGCCCAATTTTTTCAACACAAGGCTTATTGGCCAAAATATATT 3061 GCTCTAATATTTTAAAATACAAATCAAAAGAAGCGCCAACACGAGCTTGCAGACGTATTA 3121 TATATTTCTTTAACCACTCATGATTCGGATTCAACACCACCCTTAATGTACATTCGACCA 3181 ATAAATTTTCTAAATTTTCTTTATCACCAAACTGATTACCTTCTATCAATTCCATCACAC 3301 CGGTACAATAAACTTGCTCATTTGCR

Fig. 1. Nucleotide sequence of the ChfuGV protein kinase region, including 5'UTR and 3'UTR. The deduced amino acid sequence PK1 is shown in single-letter code below the respective coding $p k-1$ nucleotide sequence. The potential early and late promoter motifs CAGT and TAAG are in underlined bold. The start and stop codons are double underlined and the putative polyadenylation signals are in underlined italics. The position of the cap site is indicated with an asterisk. Potential transcriptional regulatory upstream elements are in bold [AT-rich regions, GATA, CANNTG, ATTGG, GAAC, GC-box, CAGA, and A(A/T)CGT(G/T)].

bank, located downstream of the granulin gene. This position was expected as other $p k-1$ genes in granuloviruses maintained this pattern (Wormleaton and Winstanley, 2001).

The nucleotide and the predicted amino acid sequences are indicated in Fig. 1. The initiation site (TTAATGAA) does not conform to Kozak's rules with PuNNATGPu (Kozak, 1986; Kozak, 1987). However, previous analysis detected strong translation from an initiator with a $\mathrm{T}$ in the -3 position for the late phase of baculovirus infection (Chang and Blissard, 1997). Also, other reports show that optimal baculovirus initiator resides in nucleotides at positions +4 and +5 , with a specific context 5'-ATGAa/c/gN-3' (Chang et al., 1999). The initiation site of ChfuGV $p k-1$ is in agreement with both of these findings.

An early promoter motif CAGT, adhering to consensus (T/ C)AGT of early genes in baculoviruses (Ayres et al., 1994), is present in $p k-1$ at -89 nucleotides (nt) upstream from start codon. CAGT can be present with or without a TATA box (Kogan et al., 1995). Alternatively, it has been reported that the early promoter motif can vary to be instead ACGT or even TCATT (Bischoff and Slavicek, 1994; Huang and Levin, 2001). The motif ACGT can be located at several positions such as $-24 \mathrm{nt}$ and $-99 \mathrm{nt}$ and a TCATT motif can be found at $-46 \mathrm{nt}$. Two TAAG late motifs were detected at $-63 \mathrm{nt}$ and -295 nt nucleotides upstream from the ChfuGV pk-1 start codon. Almost all late and very late baculoviral genes contain 
the TAAG promoter element with consensus (A/T/G)TAAG (Blissard and Rohrmann, 1990). The motif TAAG can act as both a promoter and as a transcription start point (Hayakawa, 1999).

Two CATT motifs were positioned at $-17 \mathrm{nt}$ and $-51 \mathrm{nt}$ from the ChfuGV $p k-1$ start codon. The mRNA start site can be located at CATT motif, as determined in many early genes such as pnk/pnl (Durantel et al., 1998), in vPK (Bischoff and Slavicek, 1994) and in ORF homologue 2L (Wormleaton and Winstanley, 2001).

Cap sites are usually found at positions between $-99 \mathrm{nt}$ to $-75 \mathrm{nt}$ with motif CAGT, that may vary slightly to CACT (Wormleaton and Winstanley, 2001). In ChfuGV pk-1, the potential cap site CAGT appears to be located at $-128 \mathrm{nt}$ from the start codon.

Five potential poly(A) signals have been detected, displaying both canonical (AATAAA) and non-canonical (ATTAAA) consensus sequences. These signals were located within the coding sequence of $p k-1$ at positions $+322 \mathrm{nt}$ and $+805 \mathrm{nt}$ downstream of the start codon and within the 3'UTR region at $+139 \mathrm{nt},+989 \mathrm{nt},+1117 \mathrm{nt}$, downstream of the stop codon. This may reflect the model of selection of alternative polyadenylation in the 3'UTR during transcription, a process found to be regulated at different developmental stages or in different cellular environments (Edwalds-Gilbert et al., 1997).

The TATA box element or "basal promoter" [with consensus, $\operatorname{TATA}(\mathrm{A} / \mathrm{T}) \mathrm{A}(\mathrm{A} / \mathrm{T})]$, is usually located at positions -45 to -20 nt relative to the transcription start site (Usheva et al., 1992; Ogbourne and Antalis, 1998; Wormleaton and Winstanley, 2001). Many baculovirus early promoters contain a functional TATA box (Blissard et al., 1992); however, late promoters differ from most RNA polymerase II promoters and do not contain DNA elements such as a TATA box. ChfuGV pk-1 appears to be TATA deficient. The $v P K$ gene in LdMNPV is also TATA-less but it seems to contain two AT-rich regions which may function as early promoter elements to stimulate expression (Bischoff and Slavicek, 1994; Acharya and Gopinathan, 2002). Similarly in ChfuGV $p k-1$, several ATrich regions have been located at various positions $(-7,-55$, $-73,-167,-282,-304,-321,-367)$ and may possibly have the same influence. In baculoviruses, examples of TATA deficient promoters include all baculovirus early bro genes (Kang et al., 1999), the DNA polymerase gene (Ohresser, 1994), and lef-1 (Passarelli and Miller, 1993). In the absence of the TATA box, transcription may be subjected to other potential regulatory elements that bind to transcription factors, located at variable distances upstream from start codon. For example, elements such as the GATA motif (consensus HGATAR) is known to bind to GATA-binding transcription factors (TFs) have been identified in lepidopteran insects (Skeiky and Iatrou, 1991; Whyatt et al., 1993). In baculoviruses, the GATA motif is found in the promoters of pe-38 (Krappa et al., 1992), gp64 (Kogan and Blissard, 1994), ORF 891 (Bah et al., 1999) and in bro genes (Kang et al., 1999). In ChfuGV $p k-1$, a GATA motif is found at position $-951 \mathrm{nt}$ upstream from start codon. Other potential regulatory elements include the E-box motif (at positions $-830,-551$, and -387 in ChfuGV pk-1) with consensus CAnnTG (Kadesch, 1993) recognized by the helix-loop-helix family of transcription factors. It is important to note that the E-box motif was reported to be required for TATA-independent transcriptional activity of the gp64 efp early promoter (Kogan et al., 1995). CCAAT boxes, with consensus motif 5'-GGCCAATCT-3', usually serve as enhancer elements (Santoro et al., 1988; Ogbourne and Antalis, 1998). The frequency of CCAAT boxes appears to be relatively higher in TATA-less promoters, particularly in the reverse ATTGG orientation (Mantovani, 1998). In ChfuGV pk-1, the inverted form ATTGG has been located at about $-255 \mathrm{nt}$. The presence of a short GC-rich stretch (GC-box enhancer), with consensus 5'-GGGCGG-3' is known to increase promoter strength, sometimes, a GC-box can contain T or A (Dickson and Friesen, 1991). GC box is an important transcriptional element that can be often found in multiple repeats (quantitative effect) in the majority of eukaryotic promoters in many species, including in AcMNPV (Dickson and Friesen, 1991; Hapgood et al., 2001). There are several GC boxes within the promoter of ChfuGV $p k-1$, including one at $-247 \mathrm{nt}$ upstream of start codon (also at -365 , $-450,-482,-493,-543,-864,-904,-923,-1050$ (nt)). There are also two CAGA motifs (-721 nt and $-854 \mathrm{nt})$ in ChfuGV $p k-1$. CAGA motifs are usually recognized by the CTC factor (Tasheva et al., 2000). Finally, an enhancer-like element with consensus sequence A(A/T)CGT(G/T) (Ayres et al., 1994) containing the core CGT motif can be located at 105 nt (AACGTT) in ChfuGV pk-1. This motif has been previously identified upstream of many early genes in AcMNPV (Dickson and Friesen, 1991)

The presence and positions of promoter motifs within all baculoviral $p k-1$ genes are indicated in Table 1 . These results reflect that baculoviral $p k-1$ genes can be generally divided into three categories, those with either early or late promoter motifs and those with both early and late. With few exceptions, the positions of the late promoter motifs seem to be similar. They are located approximately at either $-30 \mathrm{nt}$ or $-60 \mathrm{nt}$ upstream from the start codon. Almost all $p k-1$ genes appear to be TATA-less, except for most GVs, which appear to have an element resembling to a TATA box at about -160 nt upstream of the start codon.

Protein sequence analysis The alignment of ChfuGV PK-1 sequence to those of other baculoviruses (Fig. 2) reveals the most conserved regions (I-XI) involved in kinase enzymatic catalysis, as defined by Hanks et al., 1988. The subdomain I containing a flexible glycine-rich loop (positions 27-29-32 in ChfuGV) with consensus V/I/L-G-X-G-X- $\phi-G-X-V$, (where $\phi$ is usually a Tyr or Phe), is involved in ATP-positioning (Bossemeyer, 1994). It is not fully conserved in GVs, including ChfuGV (positions 27, 29-32), as its glycine residues are replaced by aspartic acid residues. NPVs, however, do contain two of the three glycines. A variance 
Table 1. Presence and positions of promoter motifs on baculoviral $p k-1$ genes

\begin{tabular}{|c|c|c|c|}
\hline & $(\mathrm{A} / \mathrm{T} / \mathrm{G}) \mathrm{TAAG}^{\mathrm{a}}$ & $(\mathrm{C} / \mathrm{T}) \mathrm{AGT}^{\mathrm{b}}$ & $\operatorname{TATA}(\mathrm{A} / \mathrm{T}) \mathrm{A}(\mathrm{A} / \mathrm{T})^{\mathrm{c}}$ \\
\hline ChfuGV & -63 & $-24,-46-89,-99,-$ & -157 \\
\hline PoGV & -63 & -116 & -156 \\
\hline AoGV & -72 & -184 & -160 \\
\hline CpGV & ----- & $-24,-46,-114,-144$ & ----- \\
\hline PxGV & -61 & $-88,-152,-181$ & ----- \\
\hline $\mathrm{XcGV}$ & -16 & $-39,-111$ & -150 \\
\hline SeNPV & -138 & ----- & ----- \\
\hline HzSNPV & ----- & $-58,-89,-121,-171$ & ---- \\
\hline HaNPV & ----- & $-23,-122,-172$ & ----- \\
\hline AfNPV & -30 & $-82,-92$ & ----- \\
\hline SINPV & ---- & $-112,-172$ & ---- \\
\hline BmNPV & -30 & -92 & ---- \\
\hline AcMNPV & -29 & -58 & -42 \\
\hline OpNPV $^{\mathrm{d}}$ & ---- & ---- & ---- \\
\hline LdMNPV & -40 & $-23,-144$ & ----- \\
\hline EpNPV $^{\mathrm{d}}$ & ---- & ----- & ---- \\
\hline McNPV & ---- & -11 & ---- \\
\hline AgNPV & -29 & ----- & ----- \\
\hline
\end{tabular}

${ }^{\mathrm{a}}$ Late promoter motif.

${ }^{b}$ Early promoter motif; may be present without a TATA box; may sometimes vary to ACGT or TCATT.

'TATA box; may be generally followed 25-35 nt downstream by initiators CA(C/G)T or CATT.

${ }^{\mathrm{d}}$ Complementary strand.

within subdomain I has also been shown in other protein kinases (Russell and Nurse, 1987; Baylis et al., 1993). Subdomain II (residue 49 in ChfuGV PK-1) is characterized by the invariant Lys (consensus motif $\mathrm{K}-\mathrm{X}-\mathrm{V} / \mathrm{I} / \mathrm{L}$ ) known to interact with the $\alpha-\beta$-phosphates of bound $\mathrm{Mg}^{2+} \mathrm{ATP}$ positioning them properly for catalysis (Taylor et al., 1993). Subdomain III (residue 60 in ChfuGV PK-1) contains the glutamic acid, involved in stabilizing the ionic interactions between ATP and the lysine residue. Both subdomains II and III are strictly conserved in all baculoviral PKs. Subdomain IV defined by either isoleucine, leucine or valine is present in all baculoviruses (residue 74 in $\mathrm{ChfuGV}$ with isoleucine). Subdomain V is the site of catalysis and exhibits heterogeneity among PKs. However, baculoviral PKs appear to share the same residues (i.e. V/L/I, M, Y, and D; residues 90, 91, 93 and 98 in ChfuGV), with few exceptions. The highly conserved subdomains VI and VII have consensus motifs D-V/I/L-X-X$\mathrm{X}-\mathrm{N}-\mathrm{V} / \mathrm{I} / \mathrm{L}$ and $\mathrm{V} / \mathrm{I} / \mathrm{L}-\mathrm{X}-\mathrm{D}-\mathrm{F}-\mathrm{G}$, respectively (Hanks et al., 1988). The aspartic acid in subdomain VI is known to serve as the catalytic base as it removes the proton to activate the incoming substrate hydroxyl (Johnson et al., 1996). The asparagine residue of subdomain VI engages in hydrogen bonding interactions that orient the catalytic base. Subdomain VII contains residues Asp, Phe, and Gly, which are part of the $\mathrm{Mg}^{2+}$ positioning loop. Precisely, the aspartic acid chelates the $\mathrm{Mg}^{2+}$ that bridges the $\beta$ and $\gamma$ phosphates of ATP and is therefore essential for both ATP binding and catalysis (Zheng et al., 1993). Similarly to other serine/threonine PKs, baculoviral
PKs contain residues D-V/L/L-K-L-E-N-I/V/L in subdomain VI (residues 139 to 145 in ChfuGV), which resemble highly to the signature motif D-L-K-P-E-N. Subdomain VII in ChfuGV (residues 156 to 160) and in other baculoviral PKs, differs slightly from consensus in that it contains a tyrosine instead of a phenylalanine. A second strong indicator of Ser/ Thr specificity is found in subdomain VIII, which plays a role in peptide substrate recognition, via the triplet Ala-Pro-Glu with consensus sequence G-T/S/P-X-X-Y/W/F-X-A/P/S-P/L$\mathrm{E}$ (residues 173 to 181 in $\mathrm{ChfuGV)}$. All reported baculoviral kinases adhere to the consensus motif by having almost entirely the identical sequence G-T-X-X-Y-X-S-P-E. The short stretch D-X-W/Y/F-A/S-X-G-V/I/L in subdomain IX is well conserved in baculoviral kinases because they all contain, with few exceptions, the sequence D-X-W-A-X-G-V (residues 193 to 199 in ChfuGV PK-1). The aspartic acid is conserved because it contributes to the positioning of the catalytic loop. Subdomain $\mathrm{X}$ is a heterogeneous region and begins at 15 residues from the end of subdomain IX. It was found to be the least conserved of the 11 subdomains in other protein kinases (Hanks et al., 1988). A few baculoviral PKs begin subdomain $\mathrm{X}$ with an aspartic acid residue, namely HzSMNPV, HaSNPV, LdMNPV, ChfuGV and PxGV. The following short sequence in LdMNPV vPK, Q-X-E-X-L-X-X-X-X-L, reported to be present in several protein kinases (Bischoff and Slavicek, 1994) is roughly shared among baculoviral PKs, except for the shared presence of glutamic acid. As for ChfuGV PK-1, it contains the partial sequence D-N-X-E-X-M-X (residues 217 


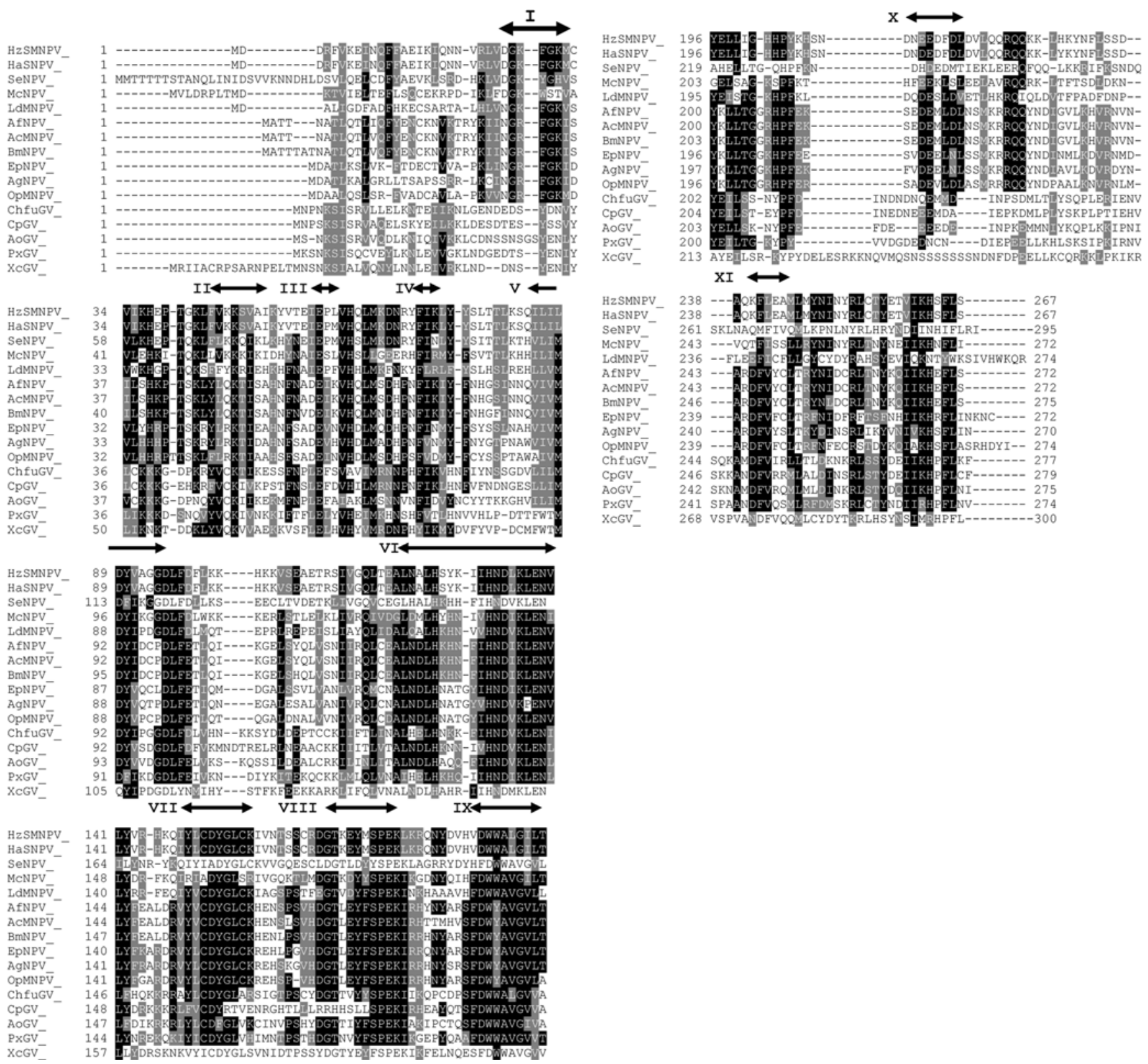

Fig. 2. Multiple alignment of deduced protein kinase amino acid sequences from ChfuGV and 15 other members of Baculoviridae family. Black indicates identity and grey indicates homology. Dashes designate gaps to allow maximum alignment. The positions of the conserved catalytic subdomains, numbered I to XI, are also indicated. Amino acid sequences were aligned using CLUSTALX (1.81). Similarity shading and scoring among the aligned sequences were determined using BOXSHADE.

to 223). Subdomain $\mathrm{XI}$ is situated 20 to 52 residues downstream of subdomain $\mathrm{X}$. It starts with an I/V/L/N/C and includes an invariant arginine at 11 residues forward, very near the carboxyl terminus of the catalytic domain. All baculoviral PKs follow the conserved pattern of subdomain XI (residues 251 and 262 in ChfuGV PK-1). ProSite predictions (Table 2) reveal several phosphorylation sites in PK-1 indicating that it may be a phosphoprotein involved in a regulatory cascade (Taylor et al., 1992). Also predicted is Nmyristoylation which is considered to be an important lipid anchor covalent modification of eukaryotic and viral proteins
(Maurer-Stroh et al., 2002), as it serves to facilitate interaction with membranes (Boutin, 1997).

The predicted molecular mass of ChfuGV PK-1 sequence stands at $32.2 \mathrm{kDa}$ adhering to the usual estimated length of the kinase catalytic core of 250-300 residues, corresponding to a protein size of approximately $30 \mathrm{kDa}$ (Hanks and Hunter, 1995). Baculoviral PKs lack the N-terminal regulatory domain. It has been previously suggested that the regulatory subunit would be the product of another viral gene (Bischoff and Slavicek, 1994).

Protein Blast results indicate that ChfuGV PK-1 has 
Table 2. Potential post-translational modifications sites in deduced amino acid sequence of ChfuGV PK-1

\begin{tabular}{lcc}
\hline \multicolumn{1}{c}{ Type of Modification } & Residues & Target sequence \\
\hline & $4-7$ & NKSI \\
$N$-glycosylation & $82-85$ & NSSG \\
& $242-245$ & NVSQ \\
Phosphorylation of protein kinase AMPc- and GMPc-dependent & $261-264$ & KRIS \\
Phosphorylation of protein kinase C & $50-52$ & TiK \\
Phosphorylation of tyrosine kinase & $38-46$ & SqK \\
$N$-myristoylation & $160-165$ & KkkDpkr \\
& $166-171$ & GlarSI \\
\end{tabular}

approximately $50 \%$ similarity within the catalytic domain to kinases involved in apoptosis (results not shown). These kinases belong to a novel subfamily named DAP-kinase related proteins, appearing to be generally pro-apoptotic, with DAP-kinase representing the prototype. DAP-kinase is a proapoptotic $\mathrm{Ca}^{2+}$ calmodulin-regulated serine/threonine kinase that participates in a wide array of apoptotic systems initiated by interferon- $\gamma$, TNF- $\alpha$, activated Fas, and detachment from extracellular matrix (Cohen and Kimchi, 2001). The catalytic domains of ChfuGV PK-1 and of DAPks appear to share high homology, as many residues likely to be important are conserved (data not shown) (Inbal et al., 2000). It is interesting to note that $\mathrm{BmN}$ cells expressed both an insect apoptosis-related gene with significant homology to the human DAP-1 gene (Okano et al., 2001), also part of the new subfamily of kinases (Levy-Strumpf and Kimchi, 1998), and a gene homologous to the iap gene of baculoviruses. Inhibition of apoptosis by IAP and P35 in baculoviruses is well documented (Bump et al., 1995; Vucic et al., 1997). Only baculoviral IE-1 has been reported so far to induce apoptosis in baculoviruses (Prikhod'ko and Miller, 1996). Whether or not ChfuGV PK-1 is pro-apoptotic remains to be determined. It is not as uncommon for a virus to induce apoptosis during infection (Hardwick, 1997) as the relationship between a virus and the host is a complex one and there may be a combination of both anti-apoptotic and pro-apoptotic activities so as to maximize viral infection.

Transcriptional analysis of the $p k-1$ gene of ChfuGV Using RT-PCR, the temporal transcriptional expression of $p k$ 1 revealed the presence of transcripts appearing as early as $2 \mathrm{~h}$ p.i. and continuing until $72 \mathrm{~h}$ p.i., suggesting it may be categorized as both an early and late baculovirus gene (Fig 3a). This correlates with the presence within the promoter of both canonical early (CAGT) and late (TAAG)- transcription start motifs. The life cycle in vitro of prototype baculovirus AcMNPV lasts up to $72 \mathrm{~h}$ p.i. for the very late phase (Pham and Sivasubramanian, 1992). If ChfuGV infection follows similar kinetics, then $p k-1$ expression until $72 \mathrm{~h}$ p.i. would lead it to be part of the occlusion phase. In LdMNPV, $v P K$ is also expressed throughout infection, whereas in AcMNPV and $\mathrm{BmNPV}$, the kinase proteins are expressed in the late and very late phases of infection. Other baculovirus genes are actively expressed throughout more than one phase of expression and are also regulated by tandem early and late promoter motifs, including gp64, pp31 and egt (Blissard and Rohrmann, 1989; Guarino and Smith, 1992, Chen et al., 1997). Northern blot hybridization using total RNA ( $\sim 40 \mu \mathrm{g}$ at $48 \mathrm{~h}$ p.i.) detected a major mRNA species of $2.4 \mathrm{~kb}$. Two other faintly visible shorter transcripts, $1.2 \mathrm{~kb}$ and $0.4 \mathrm{~kb}$, were also observed (Fig 3b). There are other examples of baculovirus genes expressed as multiple transcripts at a given time post-infection, including those of opep-2 (Shippam et al., 1997), DNA polymerase (Liu and Carstens, 1995), and ie-1 (Chisholm and Henner, 1988). This may be due to processes such as splicing, transcriptional overlapping and differential polyadenylation site usage. The appearance of multiple transcript isoforms for a single copy gene such as $p k-1$, is perhaps partly caused by multiple termination sites due the presence of several poly(A) sites.

It is most likely that $p k-1$ may be transcribed as a $1.2 \mathrm{~kb}$ transcript. The size of the transcript is in close agreement with the predicted value from the nucleotide sequence if indeed the transcript begins from one of the two late promoter motifs and terminates at position +139 from stop codon. As for the short transcript of $0.4 \mathrm{~kb}$, it may start from the either late or early promoter motifs and prematurely terminate within the coding sequence of $p k-1$, at poly(A) site located at position +233 from start codon, potentially producing a truncated form of the kinase protein, lacking almost all subdomains, except for subdomains I to V. This catalytically inactive form would probably only be capable of binding ATP. The reason for this truncated transcript isoform remains unclear. However, a truncated protein kinase, PKR, has been reported with an activity of inhibition of full-length protein kinase, in a dominant negative fashion (Abraham et al., 1998). The natural expression of truncated transcript has also been observed previously in the helicase gene of baculovirus TnGV (Bideshi et al., 1998).

The predominant transcript of $2.4 \mathrm{~kb}$ corresponds to an overlapping transcriptional unit containing transcripts of $p k-1$ and granulin with a common 5'end (results of hybridization using probe specific to granulin are not shown). This is commonly found in baculoviruses in the very late phase of 
(A)

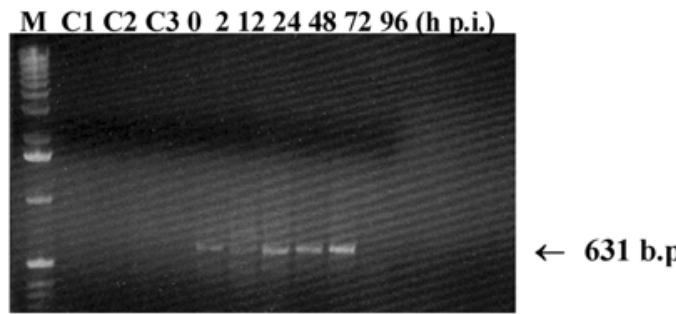

(B)

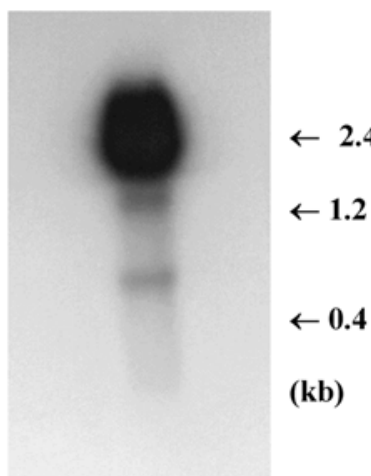

Fig. 3. (a) RT-PCR analysis: Lane headings show total RNA extracted from infected larvae at various hours post-infection. Lanes 2,3, and 4 represent the following negative controls: $\mathrm{C}$ 1: RNA from uninfected larvae, C2: no RNA, and C3: regular PCR with addition of Taq polymerase alone. (b) Northern blot on total RNA extraction from $48 \mathrm{~h}$ p.i. Membranes were blotted with a $\left[\alpha-{ }^{32} \mathrm{P}\right] \mathrm{dCTP}-$ labeled probe containing the $p k-1$ coding sequence.

infection as a means for gene regulation (Friesen and Miller, 1985; Mainprize et al., 1986). Although none of the transcripts were mapped, the transcript of $2.4 \mathrm{~kb}$ would probably begin far upstream in the granulin gene region (farther upstream at late times) and extend to include $p k-1$ transcript, terminating at a more distal polyadenylation site. The Northern blots of $v P K$ in LdMNPV reveal that polyhedrin and kinase transcripts are also co-expressed and overlapping at late times post-infection, perhaps for a regulatory purpose. (Slavicek, 1991; Bischoff and Slavicek, 1994). It is important to note that the same transcriptional pattern for ChfuGV $p k-1$ has been observed at $10 \mathrm{~h}$ p.i. and at $48 \mathrm{~h}$ p.i. (results not shown). No hybridization was detectable to RNA isolated from uninfected larvae. Phosphorylation plays an important part in baculoviral infection process as several proteins are known to be phosphorylated during the viral infection cycle. ChfuGV pk-1 being the first characterized granulovirus protein kinase, determination of the function of ChfuGV pk-1 can be considered as the next step toward a better understanding of virus/host interactions. Future experiments such as ChfuGV pk-1 expression and performing a kinase assay on the PK-1 product will be necessary to discover the nature of the phosphorylated substrate.

Acknowledgments Because of space restrictions we could not include all pertinent references and we apologize to researchers whose papers were not cited. This research was funded by the Ministère des Ressources Naturelles du Québec Grant number 0316-240S to CG.

\section{References}

Acharya, A. and Gopinathan, K. (2002) Transcriptional analysis and preliminary characterization of ORF $\mathrm{Bm} 42$ from Bombyx mori nucleopolyhedrovirus. Virology 299, 213-224.

Abraham, N., Jaramillo, M. L., Duncan, P. I., Methot, N., Icely, P. L., Stojdl, D. F., Barber, G. N. and Bell, J. C. (1998) The murine PKR tumor suppressor gene is rearranged in a lymphocytic leukemia. Exp. Cell. Res. 244, 394-404.

Altschul, S. F., Gish, W., Miller, W., Myers, E. W. and Lipman D. J. (1990) Basic local alignment search tool. J. Mol. Biol. 215, 403-410.

Ayres, M. D., Howard, S. C., Kuzio, J., Lopez-Ferber, M. and Possee, R. D. (1994) The complete DNA sequence of Autographa californica nuclear polyhedrosis virus. Virology 202, 586-605.

Bah, A., Lucarotti, C. J., Arella, M. and Guertin, C. (1999) Choristoneura fumiferana granulovirus: sequence analysis and 5' characterization of ORF891. Arch Virol. 144, 737-746.

Baylis, S. A., Banham, A. H., Vydelingum, S., Dixon, L. K. and Smith, G. L. (1993) African swine fever virus encodes a serine protein kinase which is packaged into virions. J. Virol. 67, 4549-4556.

Bideshi, D. K., Hice, R. H., Ge, B. and Federici, B. A. (1998) Molecular characterization and expression of the Trichoplusia ni granulovirus helicase gene. J. Gen. Virol. 79, 1309-1319.

Bischoff, D. S. and Slavicek, J. M. (1994) Identification and characterization of a protein kinase gene in the Lymantria dispar multinucleocapsid nuclear polyhedrosis virus. J. Virol. 68, 1728-1736.

Blissard, G. W., Ijkel, W. F., Westenberg, M., Goldbach, R. W., Blissard, G. W., Vlak, J. M. and Zuidema, D. (2000) A novel baculovirus envelope fusion protein with a proprotein convertase cleavage site. Virology 275, 30-41.

Blissard, G. W., Kogan, P. H., Wei, R. and Rohrmann, G. F. (1992) A synthetic early promoter from a baculovirus: roles of the TATA box and conserved start site CAGT sequence in basal levels of transcription. Virology 190, 783-793.

Blissard, G. W. and Rohrmann, G. F. (1990) Baculovirus diversity and molecular biology. Annu. Rev. Entomol. 35, 127-155.

Blissard, G. W. and Rorhmann, G. F. (1989) Location, sequence, transcriptional mapping, and temporal expression of the gp64 envelope glycoprotein gene of the Orgyia pseudotsugata multicapsid nuclear polyhedrosis virus. Virology 170, 537-555.

Bossemeyer, D. (1994) The glycine-rich sequence of protein kinases: a multifunctional element. Trends Biochem Sci. 19, 201-205.

Boutin, J. A. (1997) Myristoylation. Cell Signal. 9, 15-35.

Braunagel, S. C. and Summers, M. D. (1994) Autographa californica nuclear polyhedrosis virus, PDV, and ECV viral envelopes and nucleocapsids: structural proteins, antigens, lipid and fatty acid profiles. Virology 202, 315-328.

Bump, N. J., Hackett, M., Hugunin, M., Seshagiri, S., Brady, K., Chen, P., Ferenz, C., Franklin, S., Ghayur, T., Li, P., Licari, P., Mankovich, J., Shi, L., Greenberg, A. H., Miller, L. K. and 
Wong, W. W. (1995) Inhibition of ICE family proteases by baculovirus antiapoptotic protein p35. Science 269, 1885-1888.

Chang, M. J. and Blissard, G. W. (1997) Baculovirus gp64 gene expression: negative regulation by a minicistron. J. Virol. 71, 7448-7460.

Chang, M. J., Kuzio, J. and Blissard, G. W. (1999) Modulation of translational efficiency by contextual nucleotides flanking a baculovirus initiator AUG codon. Virology 259, 369-383.

Chen, X., Hu, Z., Jehle, J. A., Zhang, Y. and Vlak, J. M. (1997) Analysis of the ecdysteroid UDP-glucosyltransferase gene of Heliothis armigera single-nucleocapsid baculovirus. Virus Genes 15, 219-225.

Chisholm, G. E. and Henner, D. J. (1988) Multiple early transcripts and splicing of the Autographa californica nuclear polyhedrosis virus ie-1 gene. J. Virol. 62, 3193-3200.

Choi, J. and Guarino, L. A. (1995) Expression of the IE1 transactivator of Autographa californica nuclear polyhedrosis virus during viral infection. Virology 209, 99-107.

Chomczynski, P. and Sacchi, N. (1987) Single-step method of RNA isolation by acid guanidinium thiocyanate-phenolchloroform extraction. Anal. Biochem. 162, 156-159.

Cohen, O. and Kimchi, A. (2001) DAP-kinase: from functional gene cloning to establishment of its role in apoptosis and cancer. Cell Death Differ 8, 6-15.

Dickson, J. A. and Friesen, P. D. (1991) Identification of upstream promoter elements mediating early transcription from the 35,000-molecular-weight protein gene of Autographa californica nuclear polyhedrosis virus. J. Virol. 65, 4006-4016.

Durantel, D., Croizier, L., Ayres, M. D., Croizier, G., Possee, R. D. and Lopez-Ferber, M. (1998) The pnk/pnl gene (ORF 86) of Autographa californica nucleopolyhedrovirus is a non-essential, immediate early gene. J. Gen. Virol. 79, 629-637.

Edwalds-Gilbert, G., Veraldi, K. L. and Milcarek, C. (1997) Alternative poly(A) site selection in complex transcription units: means to an end? Nucleic Acids Res. 25, 2547-2561.

Fischer, E. H. and Krebs, E. G. (1955) Conversion of phosphorylase $\mathrm{b}$ to phosphorylase a in muscle extracts. J. Biol. Chem. 216, 121-132.

Friesen, P. D. (1997) Regulation of baculovirus early gene expression; in The Baculoviruses, Miller, L. K. (ed.), pp.141166, Plenum Press, New York, USA.

Friesen, P. D. and Miller, L. K. (1985) Temporal regulation of baculovirus RNA: overlapping early and late transcripts. $J$. Virol. 54, 392-400.

Graves, J. D. and Krebs, E. G. (1999) Protein phosphorylation and signal transduction. Pharmacol Ther. 82, 111-121.

Guarino, L. A. and Smith, M. (1992) Regulation of delayed-early gene transcription by dual TATA boxes. J. Virol. 66, 37333739.

Guarino, L. A., Dong, W., Xu, B., Boussard, D. R., Davis, R. W. and Jarvis, D. L. (1992) Baculovirus phosphoprotein pp31 is associated with virogenic stroma. J. Virol. 66, 7113-7120.

Guarino, L. A., Xu, B., Jin, J. and Dong, W. (1998) A virusencoded RNA polymerase purified from baculovirus-infected cells. J. Virol. 72, 7985-7991.

Hanks, S. K. and Hunter, T. (1995) Protein kinases 6. The eukaryotic protein kinase superfamily: kinase (catalytic) domain structure and classification. FASEB J. 9, 576-596.

Hanks, S. K., Quinn, A. M. and Hunter, T. (1988) The protein kinase family: conserved features and deduced phylogeny of the catalytic domains. Science 241, 42-52.

Hapgood, J. P., Riedemann, J. and Scherer, S. D. (2001) Regulation of gene expression by GC-rich DNA cis-elements. Cell Biol. Int. 25, 17-31.

Harwick, J. M. (1997) Virus-induced apoptosis. Adv. Pharmacol. 41, 295-336.

Hayakawa, T., Ko, R., Okano, K., Seong, S. I., Goto, C. and Maeda, S. (1999) Sequence analysis of the Xestia c-nigrum granulovirus genome. Virology 262, 277-297.

Huang, J. and Levin, D. B. (2001) Identification, transcription and sequence analysis of the Spodoptera littoralis nucleopolyhedrovirus (SpliNPV) DNA polymerase gene. Arch Virol. 146, 303-326.

Hunter, T. (2000) Signaling-2000 and beyond. Cell 100, 113-127.

Inbal, B., Shani, G., Cohen, O., Kissil, J. L. and Kimchi, A. (2000) Death-associated protein kinase-related protein 1, a novel serine/threonine kinase involved in apoptosis. Mol. Cell. Biol. 20, 1044-1054.

Jarvis, D. L. and Garcia, A. Jr. (1994) Biosynthesis and processing of the Autographa californica nuclear polyhedrosis virus gp64 protein. Virology 205, 300-313.

Johnson, L. N., Noble, M. E. and Owen, D. J. (1996) Active and inactive protein kinases: structural basis for regulation. Cell $\mathbf{8 5}$, 149-158.

Kadesch, T. (1993) Consequences of heteromeric interactions among helix-loop-helix proteins. Cell Growth Differ. 4, 49-55.

Kang, W., Suzuki, M., Zemskov, E., Okano, K. and Maeda, S. (1999) Characterization of baculovirus repeated open reading frames (bro) in Bombyx mori nucleopolyhedrovirus. J. Virol. 73, 10339-10345.

Kelly, D. C. and Lescott, T. (1984) Baculovirus replication: phosphorylation of polypeptides synthesized in Trichoplusia $n i$ neclear polyhedrosis virus-infected cells. J. Gen. Virol. 65, 1183-1191.

Kogan, P. H. and Blissard, G. W. (1994) A baculovirus gp64 early promoter is activated by host transcription factor binding to CACGTG and GATA elements. J. Virol. 68, 813-822.

Kogan, P. H., Chen, X. and Blissard, G. W. (1995) Overlapping TATA-dependent and TATA-independent early promoter activities in the baculovirus gp64 envelope fusion protein gene. J. Virol. 69, 1452-1461.

Kozak, M. (1987) An analysis of 5'-noncoding sequences from 699 vertebrate messenger RNAs. Nucleic Acids Res. 15, 81258148.

Kozak, M. (1986) Point mutations define a sequence flanking the AUG initiator codon that modulates translation by eukaryotic ribosomes. Cell 44, 283-292.

Krappa, R., Behn-Krappa, A., Jahnel, F., Doerfler, W. and KnebelMorsdorf, D. (1992) Differential factor binding at the promoter of early baculovirus gene pe 38 during viral infection: GATA motif is recognized by an insect protein. J. Virol. 66, 34943503 .

Leader, D. P. (1993) Viral protein kinases and protein phosphatases. Pharmacol. Ther. 59, 343-389.

Levy-Strumpf, N. and Kimchi, A. (1998) Death associated proteins (DAPs): from gene identification to the analysis of their apoptotic and tumor suppressive functions. Oncogene 17, 3331-3340.

Li, J. J., Kim, R. H. and Sodek, J. (1995) An inverted TATA box directs downstream transcription of the bone sialoprotein gene. 
Biochem. J. 310, 33-40.

Li, Y. and Miller, L. K. (1995) Expression and functional analysis of a baculovirus gene encoding a truncated protein kinase homolog. Virology 206, 314-323.

Liu, J. J. and Carstens, E. B. (1995) Identification, localization, transcription, and sequence analysis of the Choristoneura fumiferana nuclear polyhedrosis virus DNA polymerase gene. Virology 209, 538-549.

Lu, A. and Miller, L. K. (1997) Regulation of baculoviruses late and very late gene expression; in The baculoviruses, Miller, L. K. (ed.), pp. 193-216, Plenum Press, New York, USA.

Mainprize, T. H., Lee, K. and Miller, L. K. (1986) Variation in the temporal expression of overlapping baculovirus transcripts. Virus Res. 6, 85-99.

Mantovani, R. (1998) A survey of 178 NF-Y binding CCAAT boxes. Nucleic Acids Res. 26, 1135-1143.

Maruniak, J. E. and Summers, M. D. (1981) Autographa californica nuclear polyhedrosis virus phosphoproteins and synthesis of intracellular proteins after virus infection. Virology 109, 25-34.

Maurer-Stroh, S., Eisenhaber, B. and Eisenhaber, F. (2002) Nterminal N-myristoylation of proteins: refinement of the sequence motif and its taxon-specific differences. J. Mol. Biol. 317, 523-540.

Miller, L. K., Adang, M. J. and Browne, D. (1983) Protein kinase activity assiciated with the extracellular and occluded forms of the baculovirus Autographa californica nuclear polyhedrosis virus. J. Virol. 46, 275-278.

Ogbourne, S. and Antalis, T. M. (1998) Transcriptional control and the role of silencers in transcriptional regulation in eukaryotes. Biochem. J. 331, 1-14.

Ohresser, M., Morin, N., Cerutti, M. and Delsert, C. (1994) Temporal regulation of a complex and unconventional promoter by viral products. J. Virol. $\mathbf{6 8}, 2589-2597$.

Okano, K., Shimada, T., Mita, K. and Maeda, S. (2001) Comparative expressed-sequence tag analysis of diffential gene expression profiles in BmNPV-infected $\mathrm{BmN}$ cells. Virology 282, 348-356.

Passarelli, A. L. and Miller, L. K. (1993) Identification and characterization of $l e f-1$, a baculovirus gene involved in late and very late gene expression. J. Virol. 67, 3481-3488.

Pham, D. Q. and Sivasubramanian, N. (1992) In vivo transcriptional analysis of three baculovirus genes: evidence of homology between viral and host transcripts. Virology 190, 288-297.

Prikhod'ko, E. A. and Miller, L. K. (1996) Induction of apoptosis by baculovirus transactivator IE-1. J. Virol. 70, 7116-7124.

Rashidan, K. K., Nassoury, N., Giannopoulos, P. N. and Guertin, C. (2002) Identification and characterization of a conserved baculoviral structural protein ODVP-6E/ODV-E56 from Christoneura fumiferana granulovirus. J. Biochem. Mol. Biol. 35, 595-603.

Reilly, L. M. and Guarino, L. A. (1994) The $p k-1$ gene of Autographa californica multinucleocapsid nuclear polyhedrosis virus encodes a protein kinase. J. Gen. Virol. 75, 2999-3006.

Rohrmann, G. F. (1999) Nuclear polyhedrosis viruses; in Encyclopedia of Virology, Webster, R. G. and Granoff, A. (eds.), pp. 146-152, Academic Press, London, United Kingdom.

Rubin, G. M., Yandell, M. D., Wortman, J. R., Gabor-Miklos, G. L., Nelson, C. R., Hariharan, I. K., Fortini, M. E., Li, P. W.,
Apweiler, R., Fleischmann, W., Cherry, J. M., Henikoff, S., Skupski, M. P., Misra, S., Ashburner, M., Birney, E., Boguski, M. S., Brody, T., Brokstein, P., Celniker, S. E., Chervitz, S. A., Coates, D., Cravchik, A., Gabrielian, A., Galle, R. F., Gelbart, W. M., George, R. A., Goldstein, L. S., Gong, F., Guan, P., Harris, N. L., Hay, B. A., Hoskins, R. A., Li, J., Li, Z., Hynes, R. O., Jones, S. J., Kuehl, P. M., Lemaitre, B., Littleton, J. T., Morrison, D. K., Mungall, C., O'Farrell, P. H., Pickeral, O. K., Shue, C., Vosshall, L. B., Zhang, J., Zhao, O., Zheng, X. H. and Lewis, S. (2000) Comparative genomics of the eukaryotes. Science 287, 2204-2215.

Russell, P. and Nurse, P. (1987) Negative regulation of mitosis by wee1+, a gene encoding a protein kinase homolog. Cell 49, 559-567.

Santoro, C., Mermod, N., Andrews, P. C. and Tjian, R. (1988) A family of human CCAAT-box-binding proteins active in transcription and DNA replication: cloning and expression of multiple cDNAs. Nature 334, 218-224.

Shippam, C., Wu, X., Stewart, S. and Theilmann, D. A. (1997) Characterization of a unique OpMNPV-specific early gene not required for viral infection in tissue culture. Virology 227, 447459.

Skeiky, Y. A. and Iatrou, K. (1991) Synergistic interactions of silkmoth chorion promoter-binding factors. Mol. Cell. Biol. 11, 1954-1964.

Slavicek, J. M. (1991) Temporal analysis and spatial mapping of Lymantria dispar nuclear polyhedrosis virus transcripts and in vitro translation polypeptides. Virus Res. 20, 223-236.

Tasheva, E. S., Conrad, A. H. and Conrad, G. W. (2000) Identification and characterization of conserved cis-regulatory elements in the human keratocan gene promoter. Biochim. Biophys. Acta 1492, 452-459.

Taylor, S. S., Knighton, D. R., Zheng, J., Ten Eyck, L. F. and Sowadski, J. M. (1992) Structural framework for the protein kinase family. Annu. Rev. Cell. Biol. 8, 429-462.

Taylor, S. S., Raddzio-Andzelm, E., Knighton, D. R., Ten Eyck, L. F., Sowadski, J. M., Herberg, F. W., Yonemoto, W. and Zheng, J. (1993) Crystal structures of the catalytic subunit of cAMP-dependent protein kinase reveal general features of the protein kinase family. Receptor 3, 165-172.

Thompson, J. D., Gibson, T. J., Plewniak, F., Jeanmougin, F. and Higgins, D. G. (1997) The ClustalX windows interface: flexible strategies for multiple sequence alignment aided by quality analysis tools. Nucleic Acids Res. 24, 4876-4882.

Usheva, A., Maldonado, E., Goldring, A., Lu, H., Houbavi, C., Reiberg, D. and Aloni, Y. (1992) Specific interaction between the nonphosphorylated form of RNA polymerase II and the TATA-binding protein. Cell 69, 871-881.

Vialard, J. E. and Richardson, C. D. (1993) The 1,629-nucleotide open reading frame located downstream of the Autographa californica nuclear polyhedrosis virus polyhedrin gene encodes a nucleocapsid-associated phosphoprotein. J. Virol. 67, 58595866.

Vucic, D., Kaiser, W. J., Harvey, A. J. and Miller, L. K. (1997) Inhibition of reaper-induced apoptosis by interaction with inhibitor of apoptosis proteins (IAPs). Proc. Natl. Acad. Sci. USA 94,10183-10188.

Wang, P., Hammer, D. A. and Granados, R. R. (1997) Binding and fusion of Autographa californica nucleopolyhedrovirus to cultured insect cells. J. Gen. Virol. 78, 3081-3089. 
Whitt, M. A. and Manning, J. S. (1988) A phosphorylated 34-kDa protein and a subpopulation of polyhedrin are thiol linked to the carbohydrate layer surrounding a baculovirus occlusion body. Virology 163, 33-42.

Whyatt, D. J., deBoer, E. and Grosveld, F. (1993) The two zinc finger-like domains of GATA-1 have different DNA binding specificities. EMBO J. 12, 4993-5005.

Wilson, M. E. and Consigli, R. A. (1985) Functions of a protein kinase activity associated with purified capsids of the granulosis virus infecting Plodia interpunctella. Virology 143, 526-535.

Winstanley, D. and O'Reilly, D. R. (1999) Granuloviruses; in Encyclopedia of Virology, Webster, R. G. and Granoff, A. (eds.), pp. 140-146, Academic Press, London, United Kingdom. Winstanley, D. and Crook, N. E. (1993) Replication of Cydia pomonella granulosis virus in cell cultures. J. Gen. Virol. 74, 1599-1609.
Wormleaton, S. L. and Winstanley, D. (2001) Phylogenetic analysis of conserved genes within the ecdysteroid UDPglucosyltransferase gene region of the slow-killing Adoxophyes orana granulovirus. J. Gen. Virol. 82, 2295-2305.

Zemskov, E. A., Abramova, E. B. and Mikhailov, V. S. (1992) Induction of a novel protein kinase in pupae of the silkworm Bombyx mori after infection with nuclear polyhedrosis virus. $J$. Gen. Virol. 73, 3231-3234.

Zhang, C. X., Hu, C. and Wu, X. F. (1998) Studies on the nucleotide sequence, transcription and deletion analysis of the BmNPV protein kinase gene. Sheng Wu Hua Xue Yu Sheng Wu Wu Li Xue Bao (Shanghai). 30, 184-190.

Zheng, J., Knighton, D. R., ten Eyck, L. F., Karlsson, R., Xuong, N., Taylor, S. S. and Sowadski, J. M. (1993) Crystal structure of the catalytic subunit of cAMP-dependent protein kinase complexed with MgATP and peptide inhibitor. Biochemistry 32, 2154-2161. 